\title{
Sarkoidose der Lunge - klinische und bronchoskopische Befunde in Abhängigkeit vom radiologischen Stadium
}

\author{
J. Schildge ${ }^{1}$ \\ B. Klar $^{2}$
}

\section{Zusammenfassung}

Die Stadieneinteilung der Lungensarkoidose erfolgt nach morphologischen Kriterien der Thoraxröntgenaufnahme. In dieser Arbeit wurde untersucht, ob zwischen Patienten in den verschiedenen radiologischen Stadien Unterschiede bei demografischen, lungenfunktionellen und bronchoskopischen Befunden bestehen. Wir untersuchten ein Kollektiv von 170 Patienten mit neu diagnostizierter Sarkoidose (Stadium I/II/III: 79/39/52) mithilfe eines multinominalen logistischen Regressionsmodells zur Analyse von Wahrscheinlichkeiten. Dabei zeigte sich eine höhere Wahrscheinlichkeit für ein Stadium I bei geringerem Alter ( $p<0,001)$, bei höherer IVC und $\operatorname{FEV}_{1}(p<0,001)$, bei einem geringeren Nikotinkonsum ( $p<0,035)$, bei in der BAL geringerem Anteil an eosinophilen Granulozyten $(p=0,014)$, Mastzellen $(p=0,004)$ und CD8-Lymphozyten $(p=0,015)$ sowie bei einem geringeren Gehalt an alkalischer Phosphatase $(p=0,004)$. Für das Stadium III galten die jeweils gegenteiligen Aussagen. Mit steigender Zellzahl in der BAL nahm die Wahrscheinlichkeit des Stadiums II $z u$, die eines Stadium III ab $(p<0,013)$. Die für die Diagnose der Sarkoidose wesentlichen BAL-Eigenschaften, die Vermehrung der Lymphozyten und des Anteils der CD4-Zellen, waren zwischen den Stadien nicht verschieden. Schlussfolgernd lassen sich zwischen Patienten mit verschiedenen SarkoidoseStadien Unterschiede in demografischen und spirometrischen Befunden sowie der Art und dem Ausmaß der alveolären Entzündung nachweisen.

\section{Abstract}

The classification of pulmonary involvement in sarcoidosis is based upon the radiographic stage of disease. We investigated 170 patients with new detected sarcoidosis (stage I / II / III: 79 / 39 / 52) for differences between the stages in demographic data, lung function values and results of BAL. With a multinomial logistic regression model to estimate probabilities, we found an increased probability for stage I with lower age $(\mathrm{p}<0.001)$, higher IVC and FEV1 ( $p<0.001$ ), a less intensive smoking history $(\mathrm{p}<0.035)$, in BAL with a lower count of eosinophils $(\mathrm{p}<0.014)$, mast cells ( $p<0.004)$, CD8 lymphocytes ( $p<0.015)$ and a less content of alkaline phosphatase $(\mathrm{p}<0.004)$. For stage III the opposite results apply. With higher cell counts in BAL the probability of stage II increased, of stage III decreased ( $p<0.013$ ). The most relevant diagnostic BAL parameter in sarcoidosis, i.e. the count of lymphocytes and CD4 cells, was not different between the radiographic stages. Conclusion: Between patients with different radiographic stages of sarcoidosis there are differences in demographic data, lung function values and the pattern of alveolitis.

Institutsangaben

${ }^{1}$ Medizin. Klinik Abt. Pneumologie, St. Vincentius-Kliniken Karlsruhe (Chefarzt Dr. J. Schildge)

${ }^{2}$ Institut für Mathematische Stochastik, Universität Karlsruhe (Leiter Prof. Dr. N. Henze)

Anmerkung

Unterstützt von der Hans und Dagmar Mende Stiftung zur Förderung der Pneumologie

an den St. Vincentius-Kliniken Karlsruhe

Korrespondenzadresse

Dr. med. Johannes Schildge · St. Vincentius-Kliniken Karlsruhe gAG · Medizinische Klinik, Abteilung Pneumologie - Südendstraße $32 \cdot 76137$ Karlsruhe $\cdot$

E-mail: johannes.schildge@vincentius-ka.de·Internet: www.vincentius-ka.de

Eingang: 13. August 2004 · Nach Revision akzeptiert: 30. Juni 2005

Bibliografie

Pneumologie 2005; 59: 582-587 @ Georg Thieme Verlag KG Stuttgart · New York

DOI $10.1055 / \mathrm{s}-2005-870972$

ISSN 0934-8387 
Die Sarkoidose ist definiert als entzündliche Systemerkrankung unklarer Ursache, deren morphologisches Bild von nicht nekrotisierenden Granulomen bestimmt wird. Meist kommt es zu einer pulmonalen Manifestation. Wesentliche diagnostische Befunde sind der Nachweis von epitheloidzelligen Granulomen im bronchoskopisch entnommenen Bronchial- oder Lungengewebe und die CD4-dominierte lymphozytäre Alveolitis in der bronchoalveolären Lavage (BAL) [1].

Die Stadieneinteilung der Sarkoidose erfolgt seit 1958 nach rein morphologischen Kriterien aus der Röntgenübersichtsaufnahme des Thorax [2]:

- Stadium I: Lymphknotenvergrößerungen bihilär und mediastinal,

- Stadium II: Lymphknotenvergrößerungen und Infiltration des Lungengewebes,

- Stadium III: Infiltration des Lungengewebes ohne Lymphknotenvergrößerungen,

- Stadium IV: Fortgeschrittene Fibrose und narbige Destruktion,

Mehrere Untersuchungen gingen der Frage nach, ob das radiologische Stadium Aussagen zum Verlauf erlaubt und wie es mit weiteren Symptomen und Befunden der Sarkoidose korreliert. Dabei zeigte sich mit zunehmendem Stadium eine abnehmende Wahrscheinlichkeit spontaner Remissionen: Stadium I: 55-90\%; Stadium II: 40-70\%; Stadium III: 10-20\% [3-9]. Andererseits waren die Klinik und Lungenfunktion von Patienten in den Stadien II und III höchst variabel [10-15]. Mit fortschreitendem Röntgenstadium wurde in der BAL eine Zunahme der Neutrophilen [16,17], nicht aber der Lymphozyten [16 - 20] gesehen. Auch wurde über eine Zunahme der CD8-Lymphozyten in der BAL bei höherem Röntgenstadium berichtet [20]. Der SerumACE-Spiegel zeigte keine Korrelation mit dem radiologischen Stadium $[16,21]$.

Die vorliegende Arbeit geht der Frage nach, ob zwischen Patienten in den verschiedenen radiologischen Stadien Unterschiede bei demografischen, lungenfunktionellen und bronchoskopischen Befunden bestehen.

\section{Patienten und Methoden}

In die Auswertung wurden 170 Patienten aufgenommen, bei denen nach dem 1. Januar 1999 meist unter ambulanten Bedingungen in der Pneumologischen Abteilung der St. Vincentius-Kliniken Karlsruhe die Diagnose einer Sarkoidose gestellt wurde. Bei keinem der Patienten war zuvor eine Sarkoidose histologisch nachgewiesen oder medikamentös behandelt worden. Röntgenstadien, demografische Befunde, Raucheranamnese, Spirometrie und Resultate der BAL zum Zeitpunkt der Diagnosestellung gehen aus Tab. 1 hervor. Sämtliche Patienten wurden aus diagnostischen Gründen im Rahmen der Abklärung klinischer und radiologischer Symptome untersucht. Hierunter befanden sich keine Patienten im Stadium IV.
Tab. 1 Mittelwerte, Standardabweichungen (SD) und p-Werte der multinominalen logistischen Regressionsmodelle (MLM) mit jeweils einem Einflussfaktor

\begin{tabular}{|c|c|c|c|c|c|}
\hline & $\begin{array}{l}\text { Sarkoidose } \\
\text { Stad. } 1\end{array}$ & $\begin{array}{l}\text { Sarkoidose } \\
\text { Stad. } 2\end{array}$ & $\begin{array}{l}\text { Sarkoidose } \\
\text { Stad. } 3\end{array}$ & $\begin{array}{l}\text { Sarkoidose } \\
\text { alle }\end{array}$ & $\begin{array}{l}P \\
M L M\end{array}$ \\
\hline$N()$ & 79 (29) & $39(16)$ & $52(29)$ & $170(74)$ & \\
\hline $\begin{array}{l}\text { Alter } \\
\text { (Jahre) }\end{array}$ & $\begin{array}{l}41,1 \\
\text { SD } 13,2\end{array}$ & $\begin{array}{l}40,5 \\
\text { SD } 12,8\end{array}$ & $\begin{array}{l}49,7 \\
\text { SD } 13,9\end{array}$ & $\begin{array}{l}43,6 \\
\text { SD } 13,9\end{array}$ & $<0,001$ \\
\hline $\begin{array}{l}\text { Raucher- } \\
\text { anamnese } \\
\text { (Packyears) }\end{array}$ & $\begin{array}{l}3,5 \\
\text { SD 7,6 }\end{array}$ & $\begin{array}{l}8,2 \\
\text { SD } 13,0\end{array}$ & $\begin{array}{l}8,6 \\
\text { SD } 18,1\end{array}$ & $\begin{array}{l}6,1 \\
\text { SD } 13,0\end{array}$ & 0,035 \\
\hline IVC \%soll & $\begin{array}{l}94,7 \\
\text { SD } 15,5\end{array}$ & $\begin{array}{l}83,8 \\
\text { SD } 13,9\end{array}$ & $\begin{array}{l}80,4 \\
\text { SD } 16,8\end{array}$ & $\begin{array}{l}87,9 \\
\text { SD } 16,8\end{array}$ & $<0,001$ \\
\hline $\mathrm{FEV}_{1} \%$ soll & $\begin{array}{l}95,3 \\
\text { SD } 15,6\end{array}$ & $\begin{array}{l}83,9 \\
\text { SD } 13,2\end{array}$ & $\begin{array}{l}78,6 \\
\text { SD } 18,7\end{array}$ & $\begin{array}{l}87,7 \\
\text { SD } 17,8\end{array}$ & $<0,001$ \\
\hline $\begin{array}{l}\text { Rückgewinn } \\
\text { BAL (ml) }\end{array}$ & $\begin{array}{l}62,8 \\
\text { SD 9,7 }\end{array}$ & $\begin{array}{l}63,7 \\
\text { SD 9,0 }\end{array}$ & $\begin{array}{l}59,6 \\
\text { SD } 11,9\end{array}$ & $\begin{array}{l}62,0 \\
\text { SD } 10,4\end{array}$ & 0,12 \\
\hline $\begin{array}{l}\text { Gesamt-EW } \\
\text { BAL }(\mathrm{mg} / \mathrm{dl})\end{array}$ & $\begin{array}{l}33,3 \\
\text { SD } 49,9\end{array}$ & $\begin{array}{l}53,9 \\
\text { SD } 54,9\end{array}$ & $\begin{array}{l}30,7 \\
\text { SD } 33,2\end{array}$ & $\begin{array}{l}34,9 \\
\text { SD } 46,7\end{array}$ & 0,41 \\
\hline $\begin{array}{l}\text { Mikroalbumin } \\
\text { BAL (mg/dl) }\end{array}$ & $\begin{array}{l}14,4 \\
\text { SD } 16,9\end{array}$ & $\begin{array}{l}19,2 \\
\text { SD } 22,5\end{array}$ & $\begin{array}{l}12,1 \\
\text { SD 9,4 }\end{array}$ & $\begin{array}{l}14,8 \\
\text { SD } 16,6\end{array}$ & 0,15 \\
\hline $\begin{array}{l}A P \\
B A L(U / I)\end{array}$ & $\begin{array}{l}12,6 \\
\text { SD } 12,1\end{array}$ & $\begin{array}{l}15,7 \\
\text { SD } 16,5\end{array}$ & $\begin{array}{l}17,5 \\
\text { SD } 11,2\end{array}$ & $\begin{array}{l}14,8 \\
\text { SD } 13,1\end{array}$ & 0,004 \\
\hline $\begin{array}{l}\text { Zellen } \\
\text { BAL }\left(10^{6}\right)\end{array}$ & $\begin{array}{l}17,4 \\
\text { SD 9,8 }\end{array}$ & $\begin{array}{l}20,5 \\
\text { SD } 11,5\end{array}$ & $\begin{array}{l}14,2 \\
\text { SD 9,4 }\end{array}$ & $\begin{array}{l}17,2 \\
\text { SD } 10,3\end{array}$ & 0,013 \\
\hline $\begin{array}{l}\text { Makrophagen } \\
\text { BAL (\%) }\end{array}$ & $\begin{array}{l}63,5 \\
\text { SD } 20,5\end{array}$ & $\begin{array}{l}64,6 \\
\text { SD } 18,9\end{array}$ & $\begin{array}{l}65,3 \\
\text { SD } 21,1\end{array}$ & $\begin{array}{l}64,3 \\
\text { SD } 20,3\end{array}$ & 0,88 \\
\hline $\begin{array}{l}\text { Lymphozyten } \\
\text { BAL (\%) }\end{array}$ & $\begin{array}{l}35,2 \\
\text { SD } 20,2\end{array}$ & $\begin{array}{l}33,5 \\
\text { SD } 18,5\end{array}$ & $\begin{array}{l}32,2 \\
\text { SD 20,7 }\end{array}$ & $\begin{array}{l}33,9 \\
\text { SD } 19,9\end{array}$ & 0,69 \\
\hline $\begin{array}{l}\text { CD3 } \\
\text { (\% Lymphozyten) }\end{array}$ & $\begin{array}{l}94,2 \\
\text { SD 3,9 }\end{array}$ & $\begin{array}{l}93,3 \\
\text { SD } 4,5\end{array}$ & $\begin{array}{l}94,0 \\
\text { SD } 6,0\end{array}$ & $\begin{array}{l}94,0 \\
\text { SD 4,6 }\end{array}$ & 0,73 \\
\hline $\begin{array}{l}\text { CD4 } \\
\text { (\% Lymphozyten) }\end{array}$ & $\begin{array}{l}76,5 \\
\text { SD } 13,0\end{array}$ & $\begin{array}{l}76,8 \\
\text { SD 9,4 }\end{array}$ & $\begin{array}{l}71,6 \\
\text { SD 19,9 }\end{array}$ & $\begin{array}{l}75,2 \\
\text { SD } 13,5\end{array}$ & 0,21 \\
\hline $\begin{array}{l}\text { CD8 } \\
\text { (\% Lymphozyten) }\end{array}$ & $\begin{array}{l}15,3 \\
\text { SD } 10,1\end{array}$ & $\begin{array}{l}15,0 \\
\text { SD } 8,6\end{array}$ & $\begin{array}{l}22,1 \\
\text { SD } 14,4\end{array}$ & $\begin{array}{l}17,1 \\
\text { SD } 11,4\end{array}$ & 0,015 \\
\hline$C D 4 / C D 8$ & $\begin{array}{l}9,0 \\
\text { SD 9,5 }\end{array}$ & $\begin{array}{l}8,3 \\
\text { SD } 8,4\end{array}$ & $\begin{array}{l}5,4 \\
\text { SD } 4,4\end{array}$ & $\begin{array}{l}7,9 \\
\text { SD } 8,3\end{array}$ & 0,054 \\
\hline $\begin{array}{l}\text { Neutrophile } \\
\text { BAL (\%) }\end{array}$ & $\begin{array}{l}0,75 \\
\text { SD } 0,85\end{array}$ & $\begin{array}{l}0,78 \\
\text { SD } 0,65\end{array}$ & $\begin{array}{l}1,33 \\
\text { SD } 2,32\end{array}$ & $\begin{array}{l}0,93 \\
\text { SD } 1,46\end{array}$ & 0,071 \\
\hline $\begin{array}{l}\text { Eosinophile } \\
\text { BAL (\%) }\end{array}$ & $\begin{array}{l}0,41 \\
\text { SD } 0,64\end{array}$ & $\begin{array}{l}0,84 \\
\text { SD } 1,10\end{array}$ & $\begin{array}{l}0,81 \\
\text { SD } 1,19\end{array}$ & $\begin{array}{l}0,63 \\
\text { SD } 0,97\end{array}$ & 0,014 \\
\hline $\begin{array}{l}\text { Mastzellen } \\
\text { BAL (\%) }\end{array}$ & $\begin{array}{l}0,15 \\
\text { SD } 0,24\end{array}$ & $\begin{array}{l}0,24 \\
\text { SD } 0,43\end{array}$ & $\begin{array}{l}0,37 \\
\text { SD } 0,46\end{array}$ & $\begin{array}{l}0,24 \\
\text { SD } 0,37\end{array}$ & 0,004 \\
\hline
\end{tabular}

Die Stichprobenumfänge variierten je nach Einflussgröße: für CD3, CD4, CD8, CD4/CD8 waren 123 Messungen vorhanden; ansonsten lag der Stichprobenumfang zwischen 158 und 170.

\section{Bronchoskopie, BAL und Biopsie}

Die Bronchoskopie wurde mit einem flexiblen Bronchoskop (Olympus Optical Co. GmbH, Hamburg) unter pulsoxymetrischer Überwachung und Sauerstoffapplikation über Nasensonde durchgeführt. Nach intravenöser Prämedikation mit 5-7,5 mg Midazolam wurde das Bronchoskop unter lokaler Anästhesie mit $2 \%$ iger Xylocainlösung transoral eingeführt. Die BAL wurde meist aus einem Subsegment des Mittellappens entnommen, wobei über einen im Instrumentierkanal liegenden Kunststoffkatheter $100 \mathrm{ml}$ isotonische, sterile, im Wasserbad auf $37 \mathrm{C}$ erwärmte Kochsalzlösung in Portionen zu $20 \mathrm{ml}$ über eine Einmal- 
spritze aus Kunststoff instilliert und reaspiriert wurden. Ein Rückgewinn von über $30 \mathrm{ml}$ war Voraussetzung für die Aufnahme in die Auswertung.

Bei jedem Patient wurden mindestens eine transbronchiale Lungenbiopsie (TBB) und eine Biopsie aus dem zentralen Bronchialsystem entnommen. Bevorzugter Ort der TBB war der Mittellappen, jedoch nicht das lavagierte Segment. Der Nachweis von Epitheloidzellgranulomen wurde bei 159 Patienten erbracht, 11-mal wurde die histologische Diagnose aus mediastinoskopisch entnommenen Lymphknoten gestellt.

\section{BAL-Zellanalytik}

Nach Entnahme von $5 \mathrm{ml}$ zur mikrobiologischen Analytik wurde die BAL durch Filtration über Gaze (Topper 8, Fa. Johnson \& Johnson, Norderstedt) von groben Partikeln befreit und in einem sterilen Kunststoffgefäß gepoolt. Die Gesamtzellzahl wurde an einem Zellanalysegerät (K 800, Fa. Sysmex, Norderstedt) durchflusszytometrisch bestimmt. Zellpräparate wurden durch Zentrifugation (Cytospin 2, Fa. Shandon, Frankfurt a.M.) angefertigt. Die prozentuale Verteilung der Zellarten wurde durch mikroskopische Auszählung von 500 Zellen eines nach May-GrünwaldGiemsa gefärbten Präparates ermittelt. Bei einem Lymphozytenanteil von über $15 \%$ wurde die prozentuale Verteilung der Lymphozytenoberflächenmarker CD3 (T-Zellen), CD4 (T-Helfer-Zellen), CD8 (T-Suppressor-Zellen) und der CD4/CD8-Quotient bestimmt. Als Sekundärantikörper diente der Alkalische Phosphatase - Anti-Alkalische Phosphatase - Antikörper (Dako, Hamburg). Pro Marker wurde ein Zytozentrifugenpräparat gefärbt und durch Auszählung von jeweils 200 Lymphozyten der prozentuale Anteil der positiven Zellen bestimmt.

\section{BAL - nichtzelluläre Parameter}

Der Albumingehalt wurde über ein käufliches Testverfahren immunologisch durch Einsatz von Kaninchenantiserum (Fa. Dade Behring, Marburg) und anschließender vollautomatischer Messung am Nephelometer (BN 100, Fa. Dade Behring, Marburg) bestimmt.

Die alkalische Phosphatase (AP) wurde nach früherer Evaluierung im Entscheidungsbereich [24] mittels eines kommerziellen Testkits an einem Merck-Mega ${ }^{\oplus}$-Autoanalyzer (Fa. Merck/Toshiba, Darmstadt) bestimmt.

\section{Statistik}

Zur Klärung unserer Fragestellung erfolgten Analysen mithilfe von multinominalen logistischen Regressionsmodellen zur Schätzung von Wahrscheinlichkeiten.

Zunächst wurden die Merkmale einzeln, danach in ihrer Gesamtheit untersucht. Das Testverfahren erlaubt eine Aussage zu der Frage, ob die Ausprägung eines untersuchten Merkmals Einfluss auf die Wahrscheinlichkeit für das Vorliegen eines bestimmten Röntgenstadiums nimmt, wobei folgender mathematischer Ansatz zu Grunde liegt:

\section{Einzelne multinominale logistische Modelle}

Hier wird die Wahrscheinlichkeit $\mathrm{p}_{\mathrm{ij}}$, dass Patient i mit Wert $\mathrm{x}_{\mathrm{i}}$ einer bestimmten Einflussgröße $\mathrm{x}$ die Sarkoidose Stadium j hat, folgendermaßen modelliert:

$\mathrm{p}_{\mathrm{ij}}=\exp \left(\mathrm{a}_{\mathrm{j}}+\mathrm{b}_{\mathrm{j}} \mathrm{x}_{\mathrm{i}}\right) /\left[1+\exp \left(\mathrm{a}_{2}+\mathrm{b}_{2} \mathrm{x}_{\mathrm{i}}\right)+\exp \left(\mathrm{a}_{3}+\mathrm{b}_{3} \mathrm{x}_{\mathrm{i}}\right)\right], \quad \mathrm{j}=2,3$ $\mathrm{p}_{\mathrm{i} 1}=1-\mathrm{p}_{\mathrm{i} 2}-\mathrm{p}_{\mathrm{i} 3}$.

Hieraus ergibt sich

$\log \left(\mathrm{p}_{\mathrm{ij}} / \mathrm{p}_{\mathrm{i} 1}\right)=\mathrm{a}_{\mathrm{j}}+\mathrm{b}_{\mathrm{j}} \mathrm{x}_{\mathrm{i}}$,

$\mathrm{j}=2,3$,

also ein linearer Zusammenhang zwischen $\mathrm{x}$ und dem Logarithmus des Quotienten $\mathrm{p}_{2} / \mathrm{p}_{1}$ bzw. $\mathrm{p}_{3} / \mathrm{p}_{1}$. Außer dieser Annahme über den Zusammenhang zwischen Einflussgröße und Wahrscheinlichkeiten macht die multinominale logistische Regression keine weiteren Annahmen (etwa Verteilungsannahmen). Der grafische Verlauf der mit der Maximum-Likelihood-Methode geschätzten Wahrscheinlichkeiten $\mathrm{p}_{\mathrm{ij}}$ geht aus den Abbildungen hervor. Um zu testen, ob die Wahrscheinlichkeiten von einem bestimmten Merkmal x abhängen, wurden Likelihood-Quotienten-Tests zwischen einem Modell mit konstanten Wahrscheinlichkeiten und dem oben beschriebenen Modell durchgeführt. Signifikante Resultate bedeuten, dass die Parameter $b_{2}$ oder $b_{3}$ statistisch signifikant $(\mathrm{p}<0,05)$ von Null verschieden sind.

\section{Multinominales logistisches Modell mit mehreren Einflussgrößen}

Bei Einflussgrößen $\mathrm{x}_{1}, \mathrm{x}_{2}, \ldots, \mathrm{x}_{\mathrm{k}}$ gilt entsprechend

$\log \left(\mathrm{p}_{\mathrm{ij}} / \mathrm{p}_{\mathrm{i} 1}\right)=\mathrm{a}_{\mathrm{j}}+\mathrm{b}_{1 \mathrm{j}} \mathrm{x}_{\mathrm{i} 1}+\mathrm{b}_{2 \mathrm{j}} \mathrm{x}_{\mathrm{i} 2}+\ldots+\mathrm{b}_{\mathrm{kj}} \mathrm{x}_{\mathrm{ik}}$,

$\mathrm{j}=2,3$.

Hier lässt sich testen, ob die Hinzunahme eines Merkmals (bei Beibehaltung der schon aufgenommenen Merkmale) einen signifikanten Effekt besitzt. Das Ziel dieser Analyse besteht darin, eine gegenseitige Beeinflussung der jeweiligen Merkmale nachzuweisen bzw. auszuschließen.

Wegen unvollständiger Angaben konnten die Merkmale CD4 und CD8 nicht berücksichtigt werden. Der Stichprobenumfang betrug 157, davon 79 ( = 48\%) im Stadium I, 34 (=22\%) im Stadium II und 48 (=30\%) im Stadium III.

\section{Ergebnisse}

\section{Likelihood-Quotienten-Tests der multinominalen logisti- schen Modelle mit einer Einflussgröße (Tab.1, Abb.1 u. 2)}

Folgend Patientenmerkmale hatten einen Einfluss auf das Krankheitsstadium: Alter, IVC, $\mathrm{FEV}_{1}$ und Raucheranamnese. Von den BAL-Eigenschaften waren der Zellgehalt, der relative Anteil an eosinophilen Granulozyten, Mastzellen, CD8-Lymphozyten sowie der Gehalt an AP von Bedeutung.

Dabei nahm die Wahrscheinlichkeit eines Stadium I zu, wenn die Patienten jünger waren $(p<0,001)$, eine höhere IVC und $\mathrm{FEV}_{1}$ in \%Soll aufwiesen $(p<0,001)$ und weniger geraucht hatten $(\mathrm{p}<0,035)$.

Weiterhin wurde ein Stadium I wahrscheinlicher, wenn in der BAL weniger eosinophile Granulozyten $(p=0,014)$, Mastzellen $(p=0,004)$, CD8-Lymphozyten $(p=0,015)$ oder ein geringerer $\mathrm{Ge}$ halt an alkalischer Phosphatase $(\mathrm{p}=0,004)$ nachweisbar waren. 

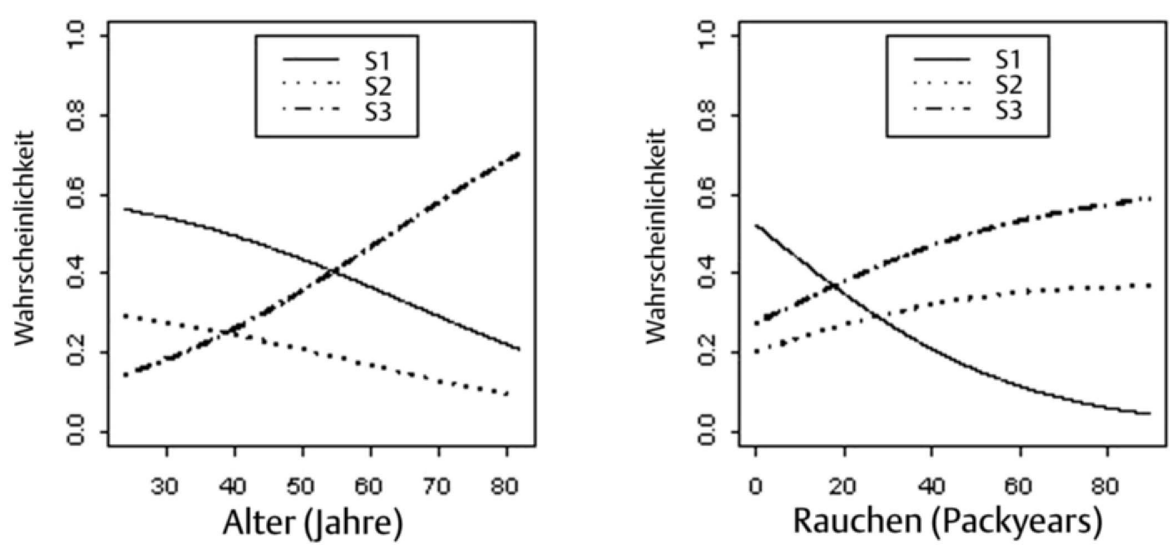

Abb. 1 Wahrscheinlichkeiten der Stadien in Abhängigkeit von der Ausprägung der signifikanten Merkmale. 0 entspricht einer Wahrscheinlichkeit von $0 \%, 1$ von $100 \%$. S1, 2, 3 = Sarkoidosestadium 1, 2, 3. IVC = inspiratorische Vitalkapazität in \% des Sollwertes; $\log \mathrm{AP}=$ Logarithmus der Konzentration der Alkalischen Phosphatase in der BAL.
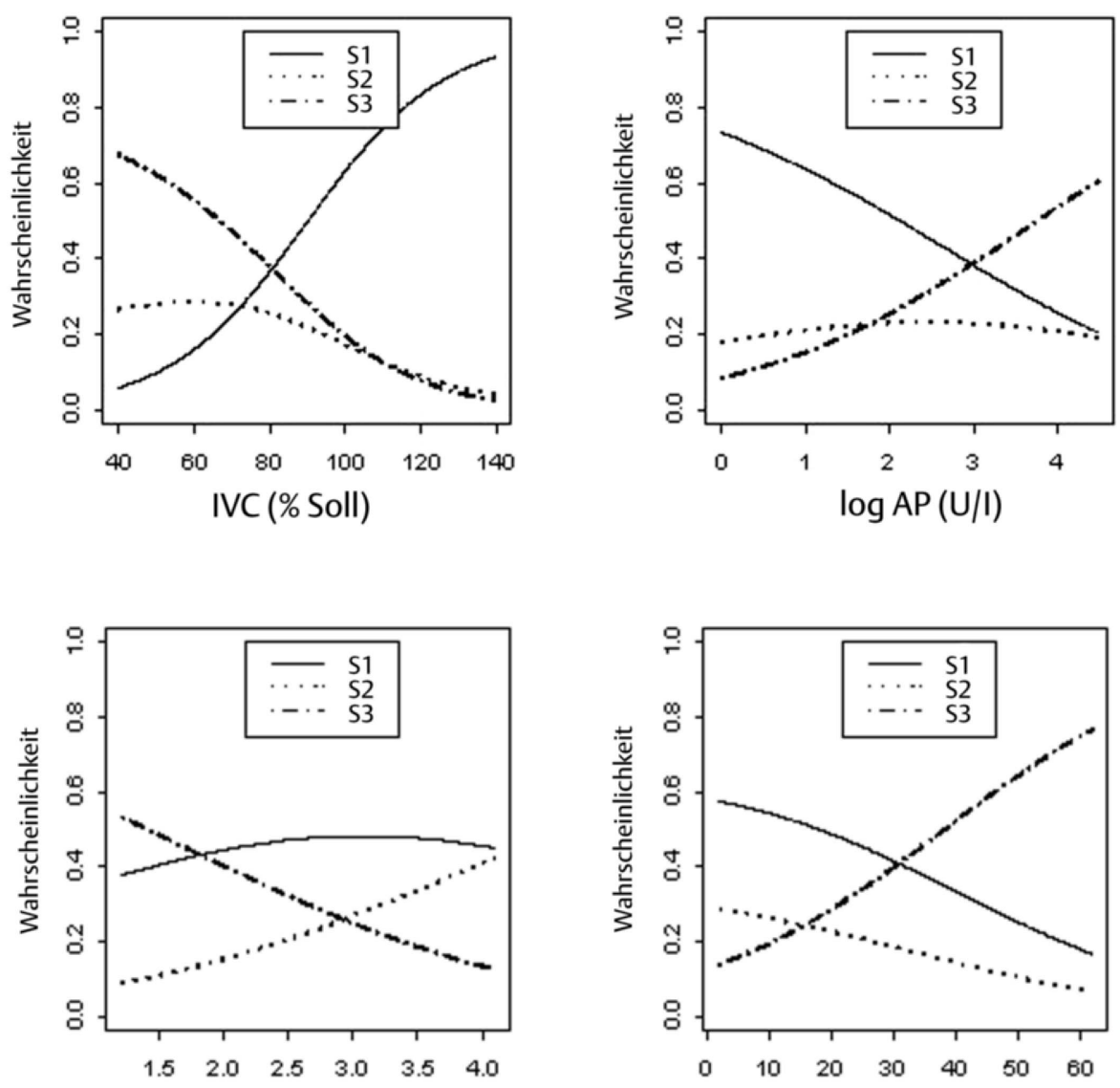

Abb. 2 Darstellungen wie Abb. 1.

CD8-Lymphozyten = Anteil der

CD8-Lymphozyten an den BAL-Lymphozyten in \%; BAL-Eosinophile = Anteil der Eosinophilen an den BAL-Zellen in \%; BALMastzellen $=$ Anteil der Mastzellen an den BAL-Zellen in \%.

log BAL-Zellzahl $\left(10^{6}\right)$
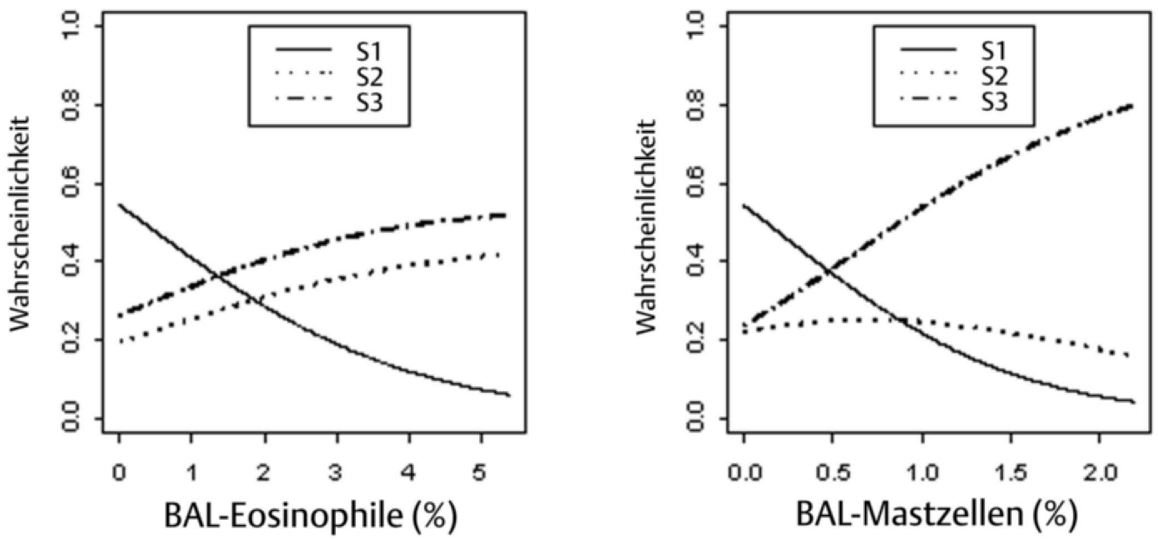
Für das Stadium III galten die jeweils gegenteiligen Aussagen verglichen mit dem Stadium I. Außerdem zeigte sich ein Trend zu einem Stadium III mit Abnahme des CD4/CD8-Quotienten $(\mathrm{p}<0,054)$.

Mit steigender Zellzahl in der BAL nahm die Wahrscheinlichkeit des Stadiums II zu, die eines Stadium III $a b(p<0,013)$. Ansonsten galt für das Stadium II, dass dessen Wahrscheinlichkeit von einer Veränderung der untersuchten Merkmale kaum beeinflusst wird.

Die für die Diagnose der Sarkoidose wesentlichen BAL-Eigenschaften, die Vermehrung der Lymphozyten und des Anteils der CD4-Zellen, waren zwischen den Stadien nicht verschieden.

\section{Multinominales logistisches Modell mit mehreren Einflussgrößen (Tab. 2)}

Bei der gemeinsamen Betrachtung der in der Einzeluntersuchung signifikanten Merkmale behalten Alter $(p=0,01)$ und IVC $(p<0,001)$ sowie die BAL-Eigenschaften Zellzahl $(p=0,01)$, Anteil der eosinophilen Granulozyten $(p=0,045)$ und der Mastzellen $(p=0,013)$ ihren Einfluss auf das Stadium. Eine wechselseitige Beziehung dieser Merkmale ist somit nicht anzunehmen. Dagegen verlieren die Merkmale Raucheranamnese und AP ihren Einfluss.

Tab. 2 p-Werte der multinominalen logistischen Regression mit mehreren Einflussfaktoren zur Analyse einer gegenseitigen Beeinflussung. Angegeben sind signifikante Abweichungen von einer konstanten Verteilung (Const) über die Stadien. „Nr.“ bezeichnet die Reihenfolge der Analysen. Dabei wird untersucht, ob die Aufnahme eines neuen Merkmales unter Beibehaltung der schon untersuchten Merkmale jeweils einen zusätzlichen signifikanten Effekt ausübt

\begin{tabular}{llll}
\hline Nr. & Modell & Test & p-Wert \\
\hline 1 & Const & & \\
\hline 2 & 1 + IVC & 1 gegen 2 & $<0,001$ \\
\hline 3 & 2+ Alter & 2 gegen 3 & 0,010 \\
\hline 4 & 3+ $\log$ (Zellen) & 3 gegen 4 & 0,001 \\
\hline 5 & 4 + Eosinophile & 4 gegen 5 & 0,045 \\
\hline 6 & 5+ Mastzellen & 5 gegen 6 & 0,013 \\
\hline
\end{tabular}

\section{Diskussion}

Die vorliegende Studie untersucht, ob sich zwischen den radiologischen Stadien der Sarkoidose Unterschiede in klinischen, funktionellen und bronchoskopischen Parametern nachweisen lassen.

Patienten im Stadium I waren im Mittel 9 Jahre jünger als im Stadium III. Dies könnte dafür sprechen, dass die radiologischen Stadien auch tatsächlich den chronologischen Ablauf der Sarkoidose widerspiegeln. Allerdings kann auch nicht ausgeschlossen werden, dass unterschiedliche, genetisch determinierte Phänotypen der Sarkoidose vorliegen, die gewisse Altersgruppen bevorzugen.
Im Röntgenstadium III ist die Vitalkapazität im Mittel um 15\% niedriger als im Stadium I. Dies spricht dafür, dass die interstitiellen Infiltrate bei Sarkoidose, die im Wesentlichen auf einer granulomatösen Entzündung beruhen, auch ohne auffällige narbige Destruktion im Sinne eines Stadium IV lungenfunktionell wirksam sind.

Die Raucheranamnese verliert bei der gemeinsamen Betrachtung aller Faktoren ihren in der Einzelanalyse signifikanten Einfluss auf das Stadium. Am ehesten ist hier eine Vermischung mit dem Faktor „Alter“ anzunehmen.

Die in der BAL nachweisbare Alveolitis unterscheidet sich zwischen den Röntgenstadien. Die höchste Gesamtzellzahl findet sich im Stadium II. Mit abnehmender Gesamtzellzahl, Zunahme von eosinophilen Granulozyten, Mastzellen und CD8-Lymphozyten wird ein Stadium III wahrscheinlicher. Der CD4/CD8-Quotient zeigt dementsprechend einen Trend zur Abnahme.

Ein Zusammenhang zwischen der im Stadium III zunehmenden Rate chronisch fibrosierender Verläufe der Sarkoidose und der beobachteten Änderung des alveolitischen Zellbildes scheint möglich. Eine Beteiligung sowohl von Mastzellen als auch von eosinophilen Granulozyten an - allerdings nicht sarkoidosebedingten - fibrotischen Umbauvorgängen des Lungengewebes $[22,23]$ wurde diskutiert.

Die gelegentlich berichtete Zunahme von neutrophilen Granulozyten im Stadium III $[16,17]$ konnten wir statistisch nicht belegen, es zeigte sich aber ein entsprechender Trend.

Möglicherweise ist dies durch das Fehlen von Patienten mit fortgeschrittener Fibrose (Stadium IV) in unserem Kollektiv zu erklären.

Die für die Diagnose einer Sarkoidose wichtigen Parameter, der Lymphozytenanteil und die CD4-Lymphozyten in der BAL, zeigen in unserer Analyse keine Unterschiede zwischen den Stadien. Dies bestätigt den in der Literatur beschriebenen fehlenden $\mathrm{Zu}$ sammenhang des Röntgenstadiums mit dem Ausmaß der lymphozytären Alveolitis [17,18]. Auch die Zunahme der CD8-Lymphozyten im Stadium III wird mitgeteilt [20].

Eine erhöhte Konzentration der AP in der BAL, welche mit einer Schädigung der Pneumozyten II in Zusammenhang stehen kann [24], korrelierte in der Einzelanalyse mit einer erhöhten Wahrscheinlichkeit eines Stadium III. Dies bestätigt einen Trend aus einer früheren Auswertung [24] und eine entsprechende Literaturmitteilung [25]. Im gemeinsamen Modell verliert sich allerdings der Einfluss der AP. Denkbar sind Einflüsse der Merkmale „Mastzellen“ und „eosinophile Granulozyten“, welche positiv mit der AP korrelieren [24].

Zusammenfassend deutet unsere Untersuchung auf Unterschiede in der Ausprägung klinischer und diagnostischer Parameter bevorzugt zwischen Patienten mit Röntgenstadium I und III der Sarkoidose hin. Ein geringeres Alter und eine weniger stark eingeschränkte Spirometrie finden sich im Stadium I. Eine Vermehrung der eosinophilen Granulozyten, der Mastzellen, der CD8-Lymphozyten und der AP in der BAL wird bevorzugt im Sta- 
dium III gesehen. Die für die Diagnose wesentlichen BAL-Befunde - das Ausmaß der lymphozytären Alveolitis und der Anteil der CD4-Lymphozyten - ist zwischen den Stadien nicht verschieden.

\section{Literatur}

1 James DG. Descriptive definition and history aspects of sarcoidosis. Clin Chest Med 1997; 18: 663-679

2 Wurm K, Reindell H, Heilmeyer L. Der Lungenboeck im Röntgenbild. Stuttgart: Thieme Verlag, 1958

${ }^{3}$ Scadding J. Prognosis of intrathoracic sarcoidosis in England: A review of 136 cases after 5 years'Observation. Br Med J 1961; 2: 1165-1172

${ }^{4}$ Siltzbach LE, James DG, Neville E. Course and prognosis of sarcoidosis around the world. Am J Med 1974; 57: 847-852

${ }^{5}$ Neville E, Walker AN, James DG. Prognostic factors predicting the outcome of sarcoidosis: An analysis of 818 patients. Q J Med 1983; 52: $525-533$

${ }^{6}$ Henke CE, Henke G, Elveback LR et al. The epidemiology of sarcoidosis in Rochester, Minnesota: A population-based study of incidence and survival. Am J Epidemiol 1986; 123: 840-845

${ }^{7}$ Lynch JP, Kazerooni EA, Gay SE. Pulmonary sarcoidosis. Clin Chest Med 1997; 18: $755-758$

${ }^{8}$ Reich JM, Johnson RE. Course and prognosis of sarcoidosis in a nonreferral setting: Analysis of 86 patients observed for 10 years. Am J Med 1985; 78: 61-67

${ }^{9}$ Chappell AG, Cheung WY, Hutchings HA. Sarcoidosis: a long-term follow up study. Sarcoidosis Vasc Diffuse Lung Dis 2000; 17: 167 - 173

${ }^{10}$ Sharma OP. Pulmonary sarcoidosis and corticosteroids. Am Rev Respir Dis 1993; 147: $1598-1600$

${ }^{11}$ Hunninghake GW, Gilbert S, Pueringer R et al. Outcome of the treatment for sarcoidosis. Am J Respir Crit Care Med 1994; 149: 893-898

${ }^{12}$ Gibson GJ, Prescott RJ, Muers MF. British Thoracic Society Sarcoidosis Study: Effects of long term corticosteroid treatment. Thorax 1996; 51: $238-247$

${ }^{13}$ Gottlieb JE, Israel HL, Steiner RM. Outcome in sarcoidosis: The relationship of relapse to corticosteroid therapy. Chest 1997; 111: 623-631
${ }^{14}$ Judson MA, Baughman RP, Thompson BW et al. Two year prognosis of sarcoidosis: The ACCESS experience. Sarcoidosis Vasc Diffuse Lung Dis 2003; 20: 204-211

${ }^{15}$ Drent M, De Vries J, Lenters M et al. Sarcoidosis: assesment of disease severity using HRCT. Eur Radiol 2003; 13: 2462 - 2471

${ }^{16}$ Drent M, Jacobs JA, De Vries J et al. Does the cellular bronchoalveolar lavage fluid profile reflect severity of sarcoidosis? Eur Respir J 1999; 13: $1338-1344$

${ }^{17}$ Arnoux A, Marsac J, Stanislav-Leguern G et al. Broncho-alveolar lavage in sarcoidosis. Correlation between alveolar lymphocytosis and clinical data. Pathol Res Pract 1982; 175: $62-79$

${ }^{18}$ Sanguinetti CM, Montroni M, Balbi B et al. Does activity of pulmonary sarcoidosis depend on disease duration? A correlation between bronchoalveolar lavage, scintigraphic, radiologic and physiologic parameters and time onset of the disease. Sarcoidosis 1987; 4: 18-24

${ }^{19}$ Israel-Biet D, Venet A, Chretien J. Persistent high alveolar lymphocytosis as a predictive criterion of chronic pulmonary sarcoidosis. Ann N Y Acad Sci 1986; 465: 395-406

${ }^{20}$ Capelli A, Di Stefano A, Lusuardi $M$ et al. Increased macrophage inflammatory protein-1alpha and macrophage inflammatory-1beta levels in bronchoalveolar lavage fluid of patients affected by different stages of pulmonary sarcoidosis. Am J Respir Crit Care Med 2002; 165: $236-241$

${ }^{21}$ Shorr AF, Torrington KG, Parker JM. Serum angiotensin converting enzyme does not correlate with radiographic stage at initial diagnosis of sarcoidosis. Respir Med 1997; 91: 399-401

22 Pesci A, Bertorelli G, Gabrielli M et al. Mast cells in fibrotic lung disorders. Chest 1993; 103: $898-896$

${ }^{23}$ Ando M, Miyazaki E, Fukami T et al. Interleukin-4-producing cells in idiopathic pulmonary fibrosis: an immunohistochemical study. Respirology 1999; 4: 883-891

${ }^{24}$ Schildge J, Klar B, Weinstock N. Wert der Bestimmung der alkalischen Phosphatase (AP) und des AP/Albuminquotienten in der bronchoalveolären Lavage für die Diagnostik interstitieller Lungenkrankheiten. Pneumologie 2000; 54: 385-391

${ }^{25}$ Capelli A, Lusuardi M, Cerutti C et al. Lung alcaline phosphatase as a marker of fibrosis in chronic interstitial disorders. Am J Respir Crit Care Med 1997; 155: 249-253 